\title{
ALKBH8-mediated formation of a novel diastereomeric pair of wobble nucleosides in mammalian tRNA
}

Erwin van den Born ${ }^{1, \star}$, Cathrine B. Vågb $\varnothing^{2, \star}$, Lene Songe-Møller ${ }^{3, \star}$, Vibeke Leihne', Guro F. Lien ${ }^{3}$, Grazyna Leszczynska ${ }^{4}$, Andrzej Malkiewicz ${ }^{4}$, Hans E. Krokan², Finn Kirpekar ${ }^{5}$, Arne Klungland ${ }^{3,6}$ \& Pål $\varnothing$. Falnes ${ }^{1,3}$

Mammals have nine different homologues (ALKBH1-9) of the Escherichia coli DNA repair demethylase AlkB. ALKBH2 is a genuine DNA repair enzyme, but the in vivo function of the other $A L K B H$ proteins has remained elusive. It was recently shown that $A L K B H 8$ contains an additional transfer RNA (tRNA) methyltransferase domain, which generates the wobble nucleoside 5-methoxycarbonylmethyluridine $\left(\mathrm{mcm}^{5} \mathrm{U}\right)$ from its precursor 5 -carboxymethyluridine $\left(\mathrm{cm}^{5} \mathrm{U}\right)$. In this study, we report that $(R)$ - and (S)-5-methoxycarbonylhydroxymethyluridine (mchm ${ }^{5} U$ ), hydroxylated forms of $\mathrm{mcm}^{5} \mathrm{U}$, are present in mammalian tRNA $\mathrm{Arg}$, and tRNA $\mathrm{GCC}^{\mathrm{Gly}}$, respectively, representing the first example of a diastereomeric pair of modified RNA nucleosides. Through in vitro and in vivo studies, we show that both diastereomers of $\mathrm{mchm}^{5} \mathrm{U}$ are generated from $\mathrm{mcm}^{5} \mathrm{U}$, and that the AlkB domain of ALKBH8 specifically hydroxylates $\mathrm{mcm}^{5} \mathrm{U}$ into (S)-mchm ${ }^{5} \mathrm{U}$ in $R_{N N A}^{G l y}$. These findings expand the function of the ALKBH oxygenases beyond nucleic acid repair and increase the current knowledge on mammalian wobble uridine modifications and their biogenesis.

\footnotetext{
${ }^{1}$ Department of Molecular Biosciences, University of Oslo, PO Box 1041 Blindern, Oslo 0316, Norway. ${ }^{2}$ Department of Cancer Research and Molecular Medicine, Norwegian University of Science and Technology, Trondheim 7489, Norway. ${ }^{3}$ Centre for Molecular Biology and Neuroscience, Department of Molecular Microbiology, Institute of Medical Microbiology, Oslo University Hospital, Oslo, Norway. ${ }^{4}$ Institute of Organic Chemistry, Technical University, Lodz 90-924, Poland. ${ }^{5}$ Department of Biochemistry and Molecular Biology, University of Southern Denmark, Odense M 5230, Denmark. ${ }^{6}$ Institute of Basic Medical Sciences, University of Oslo, PO Box 1018 Blindern, Oslo 0315, Norway. ${ }^{\star}$ These authors contributed equally to this work. Correspondence and requests for materials should be addressed to A.K. (email: arne.klungland@rr-research.no) or to P.Ø.F. (email: pal.falnes@imbv.uio.no).
} 
T ransfer RNA (tRNA) modification is important both for correct folding and for optimal interaction with components of the protein synthesis machinery, such as aminoacyl transferases, messenger RNA (mRNA) and the ribosome. The nucleotide at the wobble position in the anticodon loop of tRNA interacts with the third nucleotide of the codon in mRNA, and wobble uridines are usually modified, both in bacteria and eukaryotes ${ }^{1,2}$. Modification of wobble uridines ensures efficient decoding of both cognate and non-cognate codons $s^{3,4}$, and prevents misreading of codons from 'split' boxes, where purine- and pyrimidine-ending codons encode different amino acids ${ }^{1}$.

In eukaryotes, most wobble uridines carry the modification 5methoxycarbonylmethyluridine $\left(\mathrm{mcm}^{5} \mathrm{U}\right), 5$-carbamoylmethyluridine $\left(n \mathrm{~cm}^{5} \mathrm{U}\right)$ or derivatives thereof (Fig. 1a). These modifications and their biogenesis have been most closely studied in the yeast Saccharomyces cerevisiae. Here, tRNA isoacceptors that contain $\mathrm{ncm}^{5} \mathrm{U}$ decode in the 'family' codon boxes, in which all four codons encode the same amino acid, whereas tRNAs containing $\mathrm{mcm}^{5} \mathrm{U}$ usually decode in the split codon boxes. The Elongator complex has been shown to mediate an early step in $\mathrm{mcm}^{5} \mathrm{U} / \mathrm{ncm}^{5} \mathrm{U}$ formation ${ }^{5}$, whereas the final methyl esterification reaction leading to the formation of $\mathrm{mcm}^{5} \mathrm{U}$ is catalysed by the methyltransferase (MT) Trm9 (ref. 6).

The 2OG/Fe(II) (2-oxoglutarate- and $\mathrm{Fe}^{2+}$-dependent) oxygenase superfamily encompasses enzymes involved in important processes, for example, generation of hydroxyproline in collagen, regulation of hypoxia-responsive genes, demethylation of histone proteins, nucleic acid repair and, as discovered recently, introduction of the epigenetic modification 5-hydroxymethylcytosine $(5 \mathrm{hmC})$ in $\mathrm{DNA}^{-9}$. The a<smiles>O=c1[nH]c(=O)n(C2OC(CO)C(O)C2O)cc1P</smiles>

$\mathrm{mcm}^{5} \mathrm{U} \quad \mathrm{R}=\overbrace{\mathrm{O}}^{\mathrm{OMe}}$

$\mathrm{mchm}^{5} \mathrm{UR}=\xi \overbrace{\mathrm{O}}^{\mathrm{OH}} \mathrm{OMe}$

$\mathrm{cm}^{5} \mathrm{U} \quad \mathrm{R}=\xi \overbrace{\mathrm{O}}^{\mathrm{OH}}$

$\operatorname{chm}^{5} \mathrm{U} \quad \mathrm{R}=\overbrace{\mathrm{O}}^{\mathrm{OH}} \mathrm{OH}$

$\mathrm{ncm}^{5} \mathrm{U} \quad \mathrm{R}=\xi \overbrace{\mathrm{O}}^{\mathrm{NH}_{2}}$

$\operatorname{nchm}^{5} \cup \mathrm{R}=\overbrace{\prod_{\mathrm{O}}^{\mathrm{OH}}}^{\mathrm{OH}} \mathrm{NH}_{2}$ b

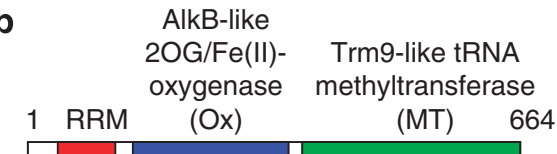

ALKBH8

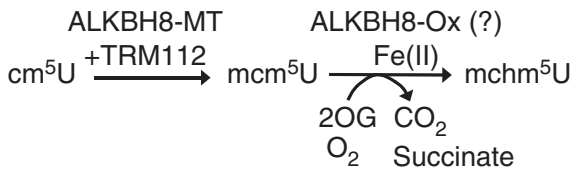

d

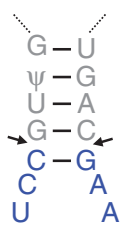

(S) $-\mathrm{mchm}^{5} \mathrm{U}_{\mathrm{C}} \mathrm{C}^{\mathrm{A}}$

tRNA $\mathrm{UCC}_{\text {Gly }}$ (B. mori)

(R)-mchm ${ }^{5} \mathrm{C}^{\mathrm{G}}$

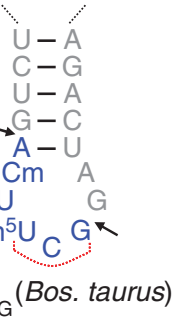

f

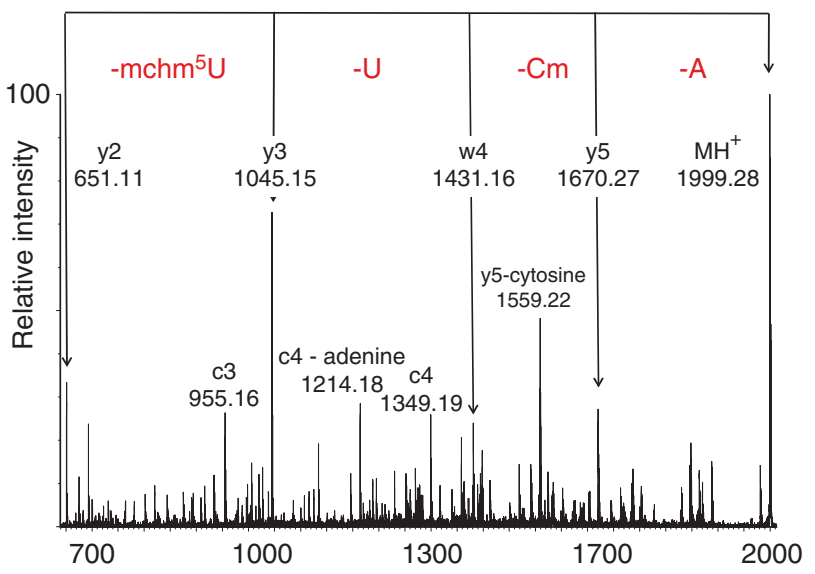

C

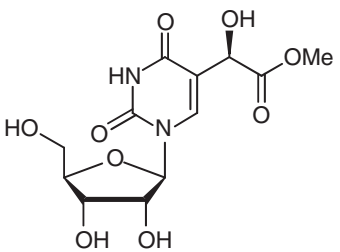

(R)-mchm 5<smiles>COC(=O)[C@H](O)c1cn(C2OC(CO)[C@@H](O)C2O)c(=O)[nH]c1=O</smiles>

(S)-mchm ${ }^{5} U$
$(R)-\mathrm{mchm}^{5} \mathrm{U}$

(S)-mchm ${ }^{5} \mathrm{U}$

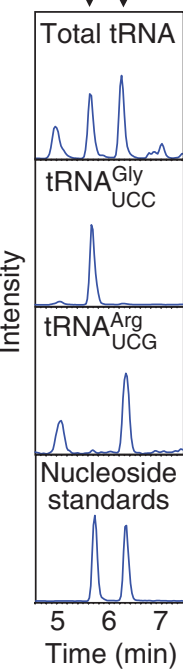

Figure 1 | Presence of two $\mathbf{m c h m}^{5} \mathbf{U}$ diastereomers in the wobble position of mammalian tRNA. (a) Wobble uridine modifications described in the present work. $\mathrm{mcm}^{5} \mathrm{U}, 5$-methoxycarbonylmethyluridine; $\mathrm{mchm}^{5} \mathrm{U}, 5$-methoxycarbonylhydroxymethyluridine; $\mathrm{chm}^{5} \mathrm{U}, 5$-carboxyhydroxymethyluridine; $\mathrm{cm}^{5} \mathrm{U}$, 5-carboxymethyluridine; $\mathrm{ncm}^{5} \mathrm{U}, 5$-carbamoylmethyluridine; $\mathrm{nchm}^{5} \mathrm{U}$, 5-carbamoylhydroxymethyluridine. (b) Domain structure and proposed function of ALKBH8. RRM, RNA recognition motif. Together with the accessory protein TRM112, the MT domain of $A L K B H 8 \mathrm{converts}^{\mathrm{cm}} \mathrm{cm}^{5}$ into $\mathrm{mcm} \mathrm{Cm}^{5} \mathrm{In}$ the present work, the $\mathrm{ALKBH} 8$ oxygenase has been investigated for its ability to hydroxylate $\mathrm{mcm}^{5} \mathrm{U}$ into $\mathrm{mchm}^{5} \mathrm{U}$. (c) Chemical structure of the two mchm ${ }^{5} \mathrm{U}$ diastereomers. (d) The anticodon stem-loop region of silkworm tRNA $\mathrm{Gly}$ and calf tRNA $\mathrm{Arg}$. Anticodon (red line) containing products (blue) generated by RNase T1 cleavage (arrows), and analysed by MALDI-TOF MS, are indicated. (e) LC-MS/MS analysis of mchm ${ }^{5}$ nucleosides in calf liver total tRNA and isolated isoacceptors. (f) MALDI-quadrupole TOF tandem mass spectrometry of the RNase T1 product harbouring the anticodon of tRNA $\mathrm{Arg}$. Fragmentation pattern suggests the sequence A-Cm-U-mchm $\mathrm{U}-\mathrm{C}-\mathrm{G}>\mathrm{p}$ as indicated. c-lons contain the original $5^{\prime}$-end after cleavage of the phophodiester bond between the phosphorous and the $5^{\prime}$-oxygen; $y$-ions contain the original $3^{\prime}$-end after cleavage of the phophodiester bond between the phosphorous and the $5^{\prime}$-oxygen. w-lons contain the original $3^{\prime}$-end after cleavage of the phophodiester bond between the $3^{\prime}$-carbon and the $3^{\prime}$-oxygen. The associated number gives the size of the fragment ion in nucleotide residues. >p denotes $2^{\prime}-3^{\prime}$ cyclic phosphate. 
2OG/Fe(II) oxygenase AlkB from Escherichia coli is a repair enzyme that can demethylate lesions in DNA and RNA, such as 1-methyladenine and 3-methylcytosine ${ }^{10-12}$. Bioinformatics analysis has identified a family of nine different mammalian AlkB homologues (ALKBH), including the obesity-associated protein $\mathrm{FTO}^{13,14}$. Although in vitro repair activities have been observed for some ALKBH proteins $s^{10,13,15,16}$, only ALKBH2 has been firmly established as a repair enzyme ${ }^{17}$, and non-repair functions have also been proposed ${ }^{18-20}$. We and others recently showed that ALKBH8 contains a MT domain, which is the functional mammalian Trm9 homologue, and requires a small accessory protein, TRM112, for activity (Fig. 1b) ${ }^{21,22}$.

As hydroxylation is the primary reaction catalysed by 2OG/ $\mathrm{Fe}(\mathrm{II})$ oxygenases in animals, and as the MT domain of ALKBH8 is involved in $\mathrm{mcm}^{5} \mathrm{U}$ biogenesis, we were intrigued by a report documenting the presence of $(S)$-5-methoxycarbonylhydroxymethyluridine $\left((S)-\mathrm{mchm}^{5} \mathrm{U}\right)$, a hydroxylated form of $\mathrm{mcm}^{5} \mathrm{U}$, in tRNA ${ }_{\mathrm{UCC}}^{\text {Gly }}$ from the silkworm Bombyx mori ${ }^{23}$ (Fig. 1c,d). Mammalian and worm tRNA $_{\text {UCC }}^{\text {Gly }}$ are highly similar, and the primary sequence of the anticodon loop is identical (Supplementary Fig. S1). This suggested that $(S)$ - $m \mathrm{Chm}^{5} \mathrm{U}$ may be present also in mammalian tRNA, and that theALKBH8oxygenasecouldberesponsibleforitsformation(Fig.1b). Indeed, whilst our manuscript was under preparation, Fu et al. independently reported that the AlkB domain of murine ALKBH8 can hydroxylate wobble $\mathrm{mcm}^{5} \mathrm{U}$ into $(S)-\mathrm{mchm}^{5} \mathrm{U}$ in a synthetic substrate resembling the anticodon stem-loop of $\mathrm{tRNA}_{\mathrm{UCC}}{ }^{24}$.

In the present work, we demonstrate that $(S)-\mathrm{mchm}^{5} \mathrm{U}$ is found in the wobble position of mammalian tRNA Gly , and that its diastereomer, $(R)-\mathrm{mchm}^{5} \mathrm{U}$, is found in $\mathrm{tRNA}_{\mathrm{UCG}}^{\mathrm{Arg}}$. Moreover, we show through studies of gene-targeted mice and recombinant enzymes that the MT domain of ALKBH8 provides the $\mathrm{mcm}^{5} \mathrm{U}$ precursor required for formation of $\mathrm{mchm}^{5} \mathrm{U}$ in tRNA $\mathrm{UCC}_{\mathrm{UCC}}^{\mathrm{Gly}}$ and $\mathrm{tRNA} \mathrm{UCG}_{\mathrm{Crg}}^{\mathrm{Arg}}$ whereas its AlkB domain represents an evolutionary conserved oxygenase that catalyses the hydroxylation of wobble $\mathrm{mcm}^{5} \mathrm{U}$ to $(S)-$ mchm $^{5} \mathrm{U}$ in tRNA $\mathrm{GCC}$

\section{Results}

Analysis of $\mathbf{m c h m}^{5} \mathrm{U}$ modifications in mammalian tRNA. To investigate the possible presence of $\mathrm{mchm}^{5} \mathrm{U}$ in mammalian tRNA, we analysed the modification status of nucleosides from bovine tRNA by liquid chromatography coupled to tandem mass spectrometry (LC-MS/MS). In this analysis, a triple-quadrupole mass spectrometer was used to specifically select the relevant nucleoside ions, induce fragmentation and detect the resulting nucleobase fragment ions. Additional specificity was obtained by comparing chromatographic retention times with those of synthetic nucleoside standards. This allows highly specific, sensitive and simultaneous quantification of several nucleosides. Intriguingly, substantial amounts of both the $S$ and $R$ diastereomers of $\mathrm{mchm}^{5} \mathrm{U}$ were detected in total bovine tRNA nucleosides (Fig. 1e). We next isolated individual, wobble uridine-containing isoacceptors by hybridization to complementary, immobilized DNA oligonucleotides. We found, as expected, $(S)-\mathrm{mchm}^{5} \mathrm{U}$ to be present in $\mathrm{tRNA}_{\mathrm{UCC}}^{\mathrm{Gly}}$, whereas tRNA ${ }_{\mathrm{UCG}}^{\mathrm{Arg}}$ contained $(R)-\mathrm{mchm}^{5} \mathrm{U}$ (Fig. 1e). We were unable to identify additional wobble uridine-containing tRNA isoacceptors carrying a $\mathrm{mchm}^{5} \mathrm{U}$ modification (data not shown). The presence of wobble $(R)-\mathrm{mchm}^{5} \mathrm{U}$ in $\mathrm{tRNA}_{\mathrm{UCG}}^{\mathrm{Arg}}$ was supported by matrixassisted laser desorption/ionization (MALDI)-time-of-flight (TOF) mass spectrometry of an RNase $\mathrm{T} 1$ digest of the purified isoacceptor. Sequencing by MALDI-quadrupole-TOF tandem mass spectrometry confirmed the presence of $\mathrm{mchm}^{5} \mathrm{U}$ at the wobble position, and the fragmentation pattern also revealed ribose methylation of C32 (ref. 25), a very common tRNA modification (Fig. 1d,f) .

Analysis of wobble uridines in Alkbh8-targeted mice. To address the possible role of the ALKBH8 oxygenase in $\mathrm{mchm}^{5} \mathrm{U}$ biogenesis, we used a mouse model, in which essential exons of the Alkbh8 gene

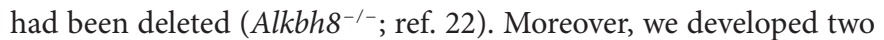
novel 'knock-in' (KI) mice in which an oxygenase-deficient Alkbh8 transgene $\left(K I\left(M T^{+}\right)\right)$or a MT-deficient Alkbh 8 transgene $\left(K I\left(O x^{+}\right)\right)$ was expressed from the Rosa26 or Hprt loci, respectively (Supplementary Figs S2-S5). These transgenes, or the combination of the two $\left(K I\left(M T^{+} / O x^{+}\right)\right)$, were introduced into the $A l k b h 8^{-/-}$background (Fig. 2a). The expression of the transgenes was verified by reverse transcription and PCR (data not shown). All the mouse models were devoid of any obvious phenotype. First, we analysed by LC-MS/MS, total tRNA nucleosides from the different mice for the presence of the two $\mathrm{mchm}^{5} \mathrm{U}$ diastereomers. As expected, wild-type (WT) tRNA contained both $(R)$ - and $(S)$ - mchm $^{5} \mathrm{U}$ (Fig. 2b). tRNA from the $A l k b h 8^{---}$and $K I\left(O x^{+}\right)$mice, which lack functional MT activity, were devoid of both $(R)$ - and $(S)-\mathrm{mchm}^{5} \mathrm{U}$, whereas the $K I\left(M T^{+}\right)$ tRNA contained exclusively $(R)-\mathrm{mchm}^{5} \mathrm{U}$. These data indicate that the formation of either $\mathrm{mchm}^{5} \mathrm{U}$ diastereomer requires the MT function, whereas the oxygenase is required for the formation of (S)-mchm ${ }^{5} \mathrm{U}$ only. This implies that a second unknown oxygenase, in the following referred to as $\mathrm{OxX}$, is responsible for the hydroxylation of $\mathrm{mcm}^{5} \mathrm{U}$ to $(R)-\mathrm{mchm}^{5} \mathrm{U}$. Furthermore, $\mathrm{KI}\left(\mathrm{MT}^{+} / \mathrm{Ox}^{+}\right)$tRNA contained, like WT tRNA, both $\mathrm{mchm}^{5} \mathrm{U}$ stereoisomers (Fig. 2b), indicating that the MT and oxygenase activities of ALKBH8 can be uncoupled and provided by two individual proteins.

To study how manipulation of ALKBH8 activity affects the formation of modified uridines in vivo, we also measured the levels of various other modifications (Fig. 2c, Supplementary Figs S6 and S7). tRNA isolated from $\mathrm{KI}\left(\mathrm{MT}^{+}\right)$mice displayed a strong accumulation of $\mathrm{mcm}^{5} \mathrm{U}$, consistent with $\mathrm{mcm}^{5} \mathrm{U}$ being a direct substrate for the ALKBH8 oxygenase. In agreement with previous results $^{22}$, we detected substantial amounts of 5-carboxymethyluridine $\left(\mathrm{cm}^{5} \mathrm{U}\right)$ and elevated levels of $\mathrm{ncm}^{5} \mathrm{U}$ in tRNA from the MTdeficient $A l k b h 8^{-1-}$ and $\mathrm{KI}\left(\mathrm{Ox}^{+}\right)$mice. Notably, we also detected 5-carbamoylhydroxymethyluridine $\left(\mathrm{nchm}^{5} \mathrm{U}\right)$ and trace amounts of 5-carboxyhydroxymethyluridine $\left(\mathrm{chm}^{5} \mathrm{U}\right)$, the hydroxylated forms of $\mathrm{ncm}^{5} \mathrm{U}$ and $\mathrm{cm}^{5} \mathrm{U}$, in tRNA from the MT-deficient mice (note that the $R$ and $S$ diastereomers of these nucleosides could not be separated chromatographically). This indicates that $\mathrm{OxX}$ and possibly also the ALKBH8 oxygenase will hydroxylate $\mathrm{ncm}^{5} \mathrm{U}$, and to a lesser extent, $\mathrm{cm}^{5} \mathrm{U}$, when $\mathrm{mcm}^{5} \mathrm{U}$ is absent.

Enzymatic activity of recombinant ALKBH8 on murine tRNA. To investigate whether the observed hypomodification of tRNA from gene-targeted mice could be reversed by recombinant enzymes, total tRNA from mouse liver was incubated with E. coli-expressed human ALKBH8, or combinations of its individual oxygenase (ALKBH8-Ox) and MT (ALKBH8-MT) domains (Fig. 2d,e). The results demonstrated that ALKBH8-Ox hydroxylates $\mathrm{mcm}^{5} \mathrm{U}$ into (S)-mchm ${ }^{5} \mathrm{U}$, and corroborated the notion that the two ALKBH8 enzymatic domains can function independently (Fig. 2e; see Fig. 2c for samples not treated with enzyme; Supplementary Fig. S8). The latter was further substantiated by the observation that the oxygenase and MT functions of ALKBH8 could be selectively inhibited by the $\mathrm{Fe}(\mathrm{II})$ chelator EDTA and the MT inhibitor S-adenosylhomocysteine, respectively (Supplementary Fig. S8). Moreover, ALKBH8Ox, but not a mutant with putative $\mathrm{Fe}(\mathrm{II})$ coordinating residues mutated, displayed the expected Fe(II)-dependent ability to convert $2 \mathrm{OG}$ to succinate (Supplementary Fig. S9), supporting the notion that ALKBH8 is a $2 \mathrm{OG} / \mathrm{Fe}(\mathrm{II})$ oxygenase.

Modification status of tRNA $\mathrm{UCC}_{\mathrm{UCC}}^{\mathrm{Gly}}$ from the Alkbh8-targeted mice. As our results strongly indicated that tRNA Gly is the substrate of the ALKBH8 oxygenase, we also specifically investigated the modification status of this isoacceptor from the different gene-targeted mice. WT tRNA $\mathrm{GCC}$ contained not only the expected $(S)-\mathrm{mchm}^{5} \mathrm{U}$ modification, but also some $\mathrm{chm}^{5} \mathrm{U}$ (Fig. 2f, Supplementary Fig. S10), possibly due to non-enzymatic hydrolysis of the labile ester 


\begin{tabular}{|c|c|c|c|c|}
\hline Mouse model & Abbreviation & Express & $\mathrm{AL}$ & protein(s \\
\hline Wild type & WT & $\begin{array}{r}\text { RRM } \\
\square\end{array}$ & $O x$ & MT \\
\hline Alkbh8 ${ }^{-/-}$ & Alkbh8 ${ }^{-/-}$ & None & & \\
\hline$A l k b h 8^{-l-} / K I\left(M T^{+}\right)$ & $K I\left(M T^{+}\right)$ & 다 & * & \\
\hline$A l k b h 8^{-I-} / K I\left(O x^{+}\right)$ & $K I\left(O x^{+}\right)$ & & & * \\
\hline $\begin{array}{c}A l k b h 8^{-1-} / K I\left(M T^{+}\right) \\
/ K I\left(O x^{+}\right)\end{array}$ & $K I\left(M T^{+} / O x^{+}\right)$ & c & & \\
\hline
\end{tabular}
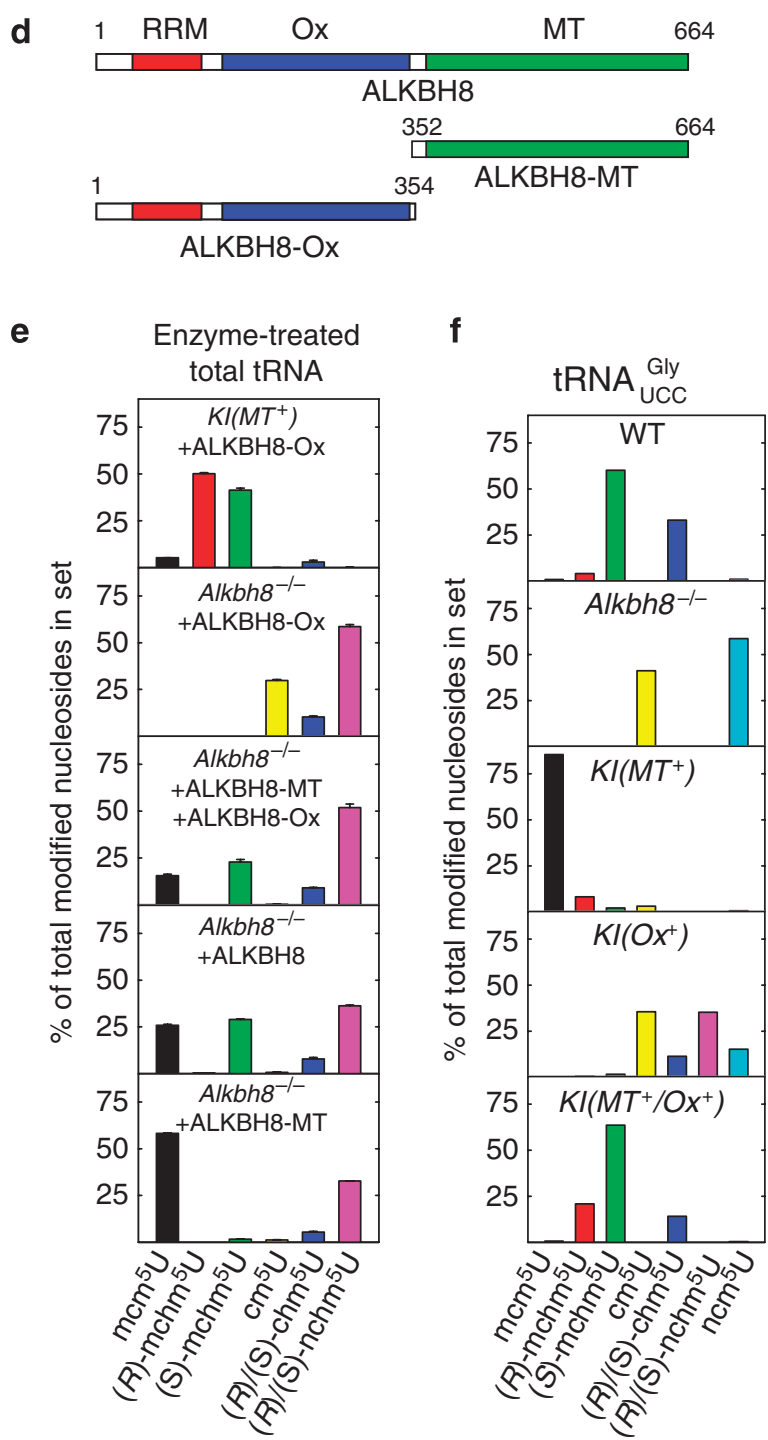

b

C

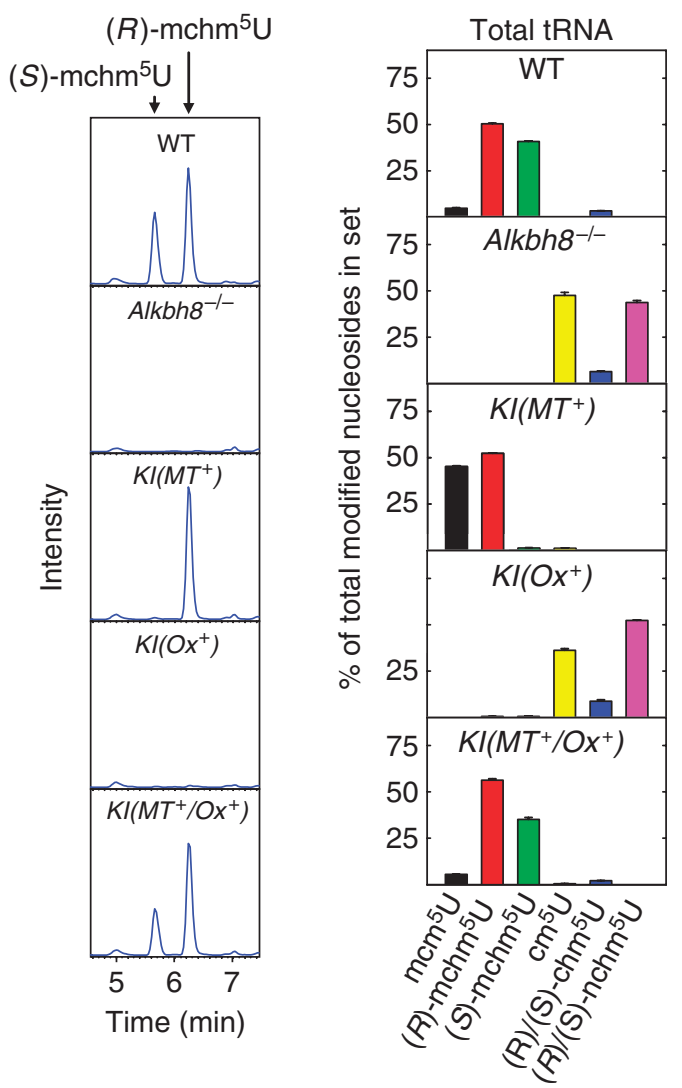

g $n \mathrm{~cm}^{5} \mathrm{U} \quad \mathrm{cm}^{5} \mathrm{U} \quad \mathrm{mcm}^{5} \mathrm{U} \quad \mathrm{nchm} \mathrm{m}^{5} \mathrm{chm} \mathrm{m}^{5} \mathrm{U} \quad \mathrm{mchm}^{5} \mathrm{U}$ $(2893.39) \quad(2894.38) \quad(2908.39)(2909.39)(2910.37)(2924.39)$

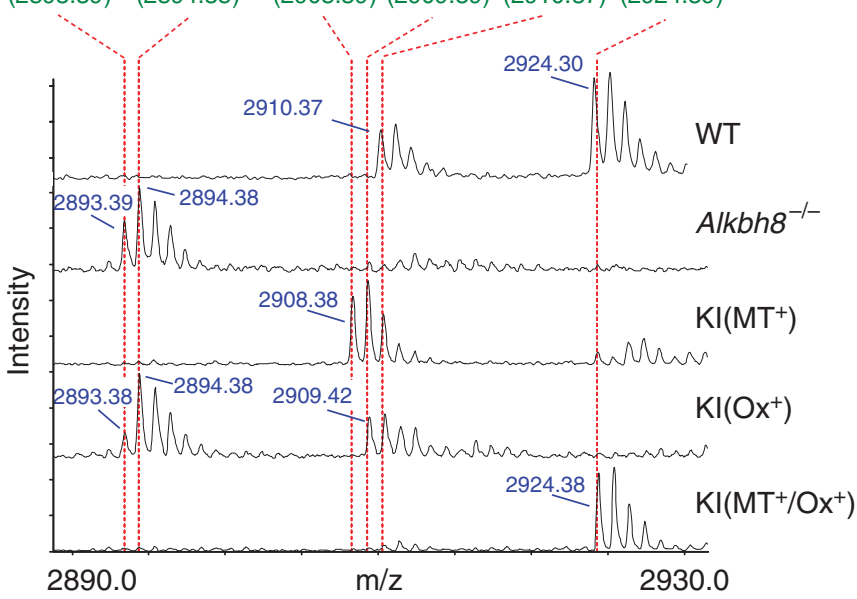

Figure 2 | Modification status of tRNA from Alkbh8-targeted mice. (a) Schematic representation of the ALKBH8 proteins expressed in the different mouse models. An asterisk indicates that the domain has been inactivated by point mutations. (b) Analysis of $R$ and $S$ diastereomers of mchm $\mathrm{U}^{5}$ in total tRNA from gene-targeted mice. Murine tRNA was enzymatically degraded to nucleosides, which were analysed by LC-MS/MS. (c) Quantification of uridine modifications in total murine tRNA by LC-MS/MS. The level of each nucleoside is expressed as the molar percentage of the total amount of the modifications indicated in the figure. (d) Recombinant, E. coli-expressed ALKBH8 proteins, or individual domains, used for incubation with murine tRNA. (e) Modification status of murine tRNA treated with human ALKBH8 or its individual domains. ALKBH8 and ALKBH8-MT were associated with co-expressed, co-purified TRM112. (f) Modification status of murine tRNA Gly measured by LC-MS/MS. (g) MALDI-TOF analysis of the anticodoncontaining RNase T1 product of tRNA GIy . The theoretical monoisotopic mass (green) as well as the measured mass (blue) of relevant modifications are indicated. Because of the natural isotope distribution, each modification gives rise to a cluster of peaks with 1.0 Da spacing where the leftmost peak corresponds to the monoisotopic mass. Overlapping isotope clusters from $\mathrm{ncm}^{5} \mathrm{U}$ and $\mathrm{cm}^{5} \mathrm{U}$ with clearly distorted peak patterns occur in the $A / k b h 8^{-/-}$ and $\mathrm{KI}\left(\mathrm{Ox}^{+}\right)$samples. Error bars represent range between duplicate samples in a typical experiment. 
bond in $(S)-\mathrm{mchm}^{5} \mathrm{U}$, as previously reported ${ }^{23}$. In agreement with

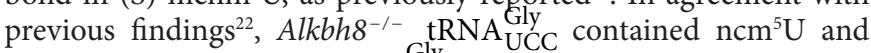
$\mathrm{cm}^{5} \mathrm{U}$, whereas $\mathrm{KI}\left(\mathrm{Ox}{ }^{+}\right)$tRNA $\mathrm{Gly}$ contained $\mathrm{nchm}^{5} \mathrm{U}$ and $\mathrm{chm}^{5} \mathrm{U}$ in addition, indicating that ALKBH8-Ox, in the absence of its cognate $\mathrm{mcm}^{5} \mathrm{U}$ substrate can hydroxylate $\mathrm{cm}^{5} \mathrm{U}$ and $n \mathrm{~cm}^{5} \mathrm{U}$, albeit inefficiently. Moreover, $K I\left(M T^{+}\right)$tRNA $\mathrm{UCC}_{\mathrm{UCC}}$ displayed the expected accumulation of $\mathrm{mcm}^{5} \mathrm{U}$. We performed MALDI-TOF mass spectrometry of an RNase T1 digest of the isolated tRNA $\mathrm{UCC}_{\mathrm{UC}}$, to verify its identity and to confirm the presence of $\mathrm{mchm}^{5} \mathrm{U}$ in its anticodon loop. Clearly, WT tRNA Gly UCC contained the expected $2924.3 \mathrm{Da}$ product corresponding to a $(S)-$ mchm $^{5} \mathrm{U}$-modified anticodon loop (Figs $1 \mathrm{~d}$ and $2 \mathrm{~g}$ ). Moreover, tRNA Gly from the gene-targeted mice gave rise to alternative additional peaks, agreeing with the altered modification pattern observed by LC-MS/MS.

Evolutionary distribution of ALKBH8 and $\mathrm{mchm}^{5} \mathrm{U}$ modifications. On the basis of sequence homology searches, the ALKBH8 oxygenase appears to be present in most multicellular eukaryotes, such as mammals, bee (Apis mellifera), worm (Caenorhabditis elegans) and plant (Arabidopsis thaliana; Supplementary Fig. S11). Accordingly, we detected $(S)-\mathrm{mchm}^{5} \mathrm{U}$ in all these organisms (Fig. 3a),

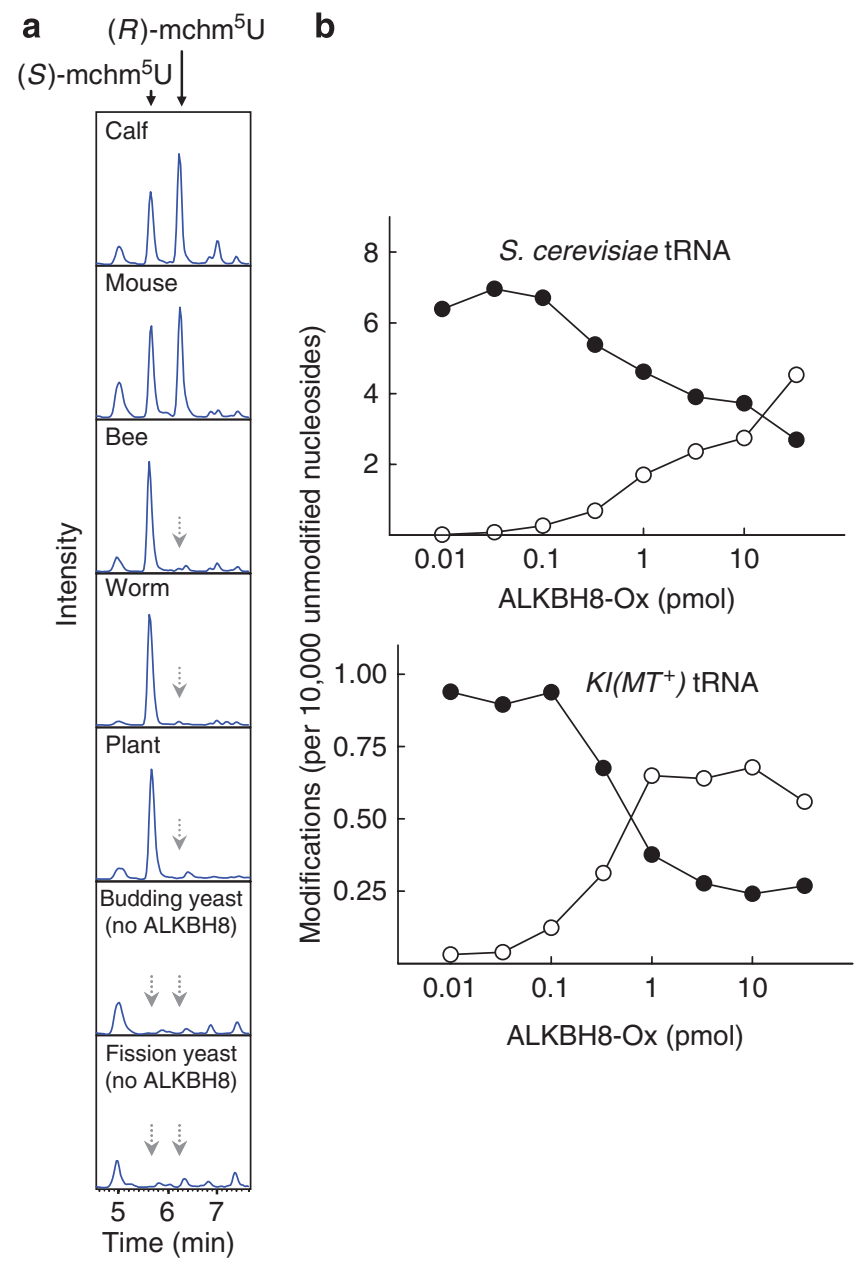

Figure 3 | Evolutionary conservation and cross-species activity of the ALKBH8 oxygenase. (a) $\mathrm{mchm}^{5} \mathrm{U}$ modifications in tRNA from various organisms, investigated by LC-MS/MS. (b) In vitro enzymatic activity of human ALKBH8-Ox on tRNA from the yeast $S$. cerevisiae (upper panel), and on tRNA from the $\mathrm{KI}\left(\mathrm{MT}^{+}\right.$) mice (lower panel). tRNA was incubated with different amounts of recombinant ALKBH8-Ox, and the levels of $\mathrm{mcm}^{5} \mathrm{U}$ (closed circles) and (S)-mchm ${ }^{5} \mathrm{U}$ (open circles) were analysed by LC-MS/MS. but neither in the budding yeast $S$. cerevisiae nor in the fission yeast Schizosaccharomyces pombe, which both have $\mathrm{mcm}^{5} \mathrm{U}$-modified tRNAs, but lack ALKBH8. Notably, $(R)-\mathrm{mchm}^{5} \mathrm{U}$ was detected only in the mammalian tissues tested (calf and mouse), and here at levels which represented $\sim 350 \mathrm{x}$ the detection limit $(0.003(R)$ mchm ${ }^{5} \mathrm{U}$ per 10,000 unmodified nucleosides). This indicates that if $(R)-\mathrm{mchm}^{5} \mathrm{U}$ is present in any of the other organisms, the level is at least two orders of magnitude lower than that in mammals. As was observed with the $\mathrm{mcm}^{5} \mathrm{U}$-containing $\mathrm{KI}\left(M T^{+}\right)$tRNA, the majority of $\mathrm{mcm}^{5} \mathrm{U}$ in $S$. cerevisiae or $S$. pombe tRNA could be converted to $(S)-\mathrm{mchm}^{5} \mathrm{U}$ by incubation with ALKBH8-Ox (Fig. 3b, Supplementary Fig. S12). Taken together, the observed activity of human ALKBH8-Ox on tRNA from distantly related organisms and the co-occurrence of $(S)-\mathrm{mchm}^{5} \mathrm{U}$ and ALKBH8 suggest that hydroxylation of $\mathrm{mcm}^{5} \mathrm{U}$ into $(S)-\mathrm{mchm}^{5} \mathrm{U}$ is performed by ALKBH8 orthologues in a wide range of eukaryotes.

Wobble uridine modifications and protein translation. In yeast, inactivation of wobble uridine modification enzymes can impair translational decoding, as measured by decreased translation of relevant 'codon-runs' ${ }^{26,27}$. To investigate the possible effects of the

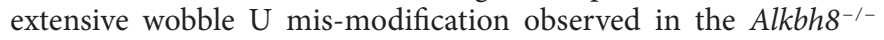
mice, we generated reporter plasmids containing both Renilla (Rluc; internal reference) and firefly (Fluc) luciferase genes, in which Fluc, but not Rluc, translation depends on in-frame translation with a codon-run of ten identical codons (Fig. 4a). We generated runs of A-ending codons matching the anticodons of several tRNAs containing $\mathrm{mcm}^{5} \mathrm{U}$ or derivatives, and corresponding non-cognate, G-ending codon-runs as controls. We then determined the Fluc/Rluc ratio on transfection of the constructs into primary fibroblasts from

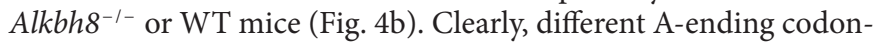
runs affected translation to varying degrees, but the differences

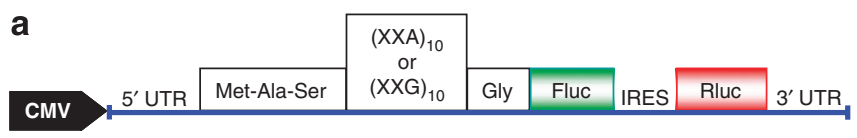

b

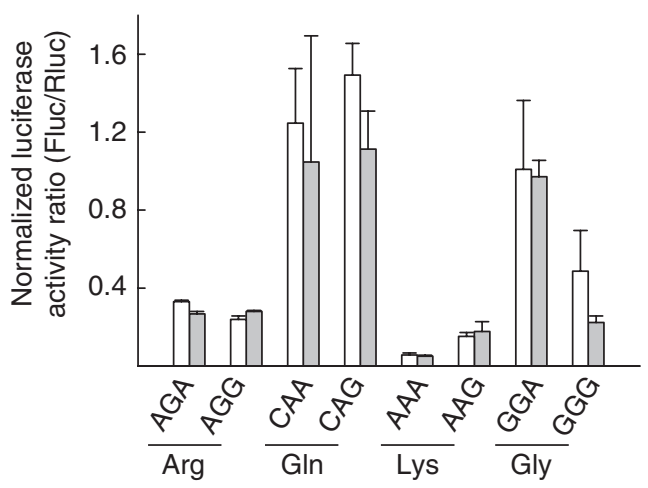

Figure 4 | Ability of Alkbh8 ${ }^{-/-}$tRNA to support translation. (a) Outline of the reporter construct used. The cytomegalovirus (CMV) promoter drives the expression of an mRNA (blue) encoding the protein elements indicated by boxes (drawing not to scale). (XXA) 10 and $(X X G)_{10}$ indicate runs of ten identical codons that end with $A$ or $G$, respectively, and that are translated in frame with the firefly luciferase (Fluc) reporter. A Renilla luciferase (Rluc) reporter, which is translated from an internal ribosomal entry site (IRES) and independently of the codon run, serves as an internal reference. (b) Reporter constructs containing the indicated codon runs, encoding the indicated amino acids, were transfected into WT (white bars) and Alkbh8 $8^{-/-}$(grey bars) fibroblasts. The activity of the two reporter genes was analysed and used to calculate the efficiency of translation of the codon run. Error bars indicate the standard deviation of triplicate samples. 


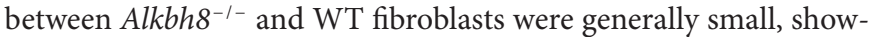
ing that the altered wobble $U$ modification pattern of $A l k b h 8^{-/-}$ tRNA has a negligible effect on translation efficiency in this experimental system.

\section{Discussion}

In this study, we have unravelled the biochemical function of the evolutionary conserved ALKBH8 oxygenase. Thus, tRNA modification is included in the broad range of reactions catalysed by $2 \mathrm{OG} / \mathrm{Fe}(\mathrm{II})$ oxygenases, and the function of the ALKBH proteins is expanded beyond nucleic acid repair. Our data demonstrate how the MT and oxygenase activities of ALKBH8 act in a sequential manner, where the MT generates $\mathrm{mcm}^{5} \mathrm{U}$, which in $\mathrm{tRNA}_{\mathrm{UCC}}^{\mathrm{Gly}}$ is further hydroxylated to $(S)-\mathrm{mchm}^{5} \mathrm{U}$ by the oxygenase. Furthermore, it is demonstrated how selective inactivation of the individual domains of ALKBH8 in vivo severely distorts the modification pattern of wobble uridines, and how the ALKBH8 oxygenase, in the absence of its cognate substrate $m c m^{5} U$, will hydroxylate $n \mathrm{~cm}^{5} \mathrm{U}$ and $\mathrm{cm}^{5} \mathrm{U}$, albeit inefficiently (Fig. 5).

The present study reports the existence of two novel mammalian wobble uridine modifications, $(R)$ - and $(S)$ - $\mathrm{mchm}^{5} \mathrm{U}$, and indicates that the evolution of more advanced eukaryotes has been accompanied by an increased complexity of such modifications. Most $\mathrm{mcm}^{5} \mathrm{U}$-containing wobble nucleosides contain an additional, wobble restricting 2-thiolation $\left(\mathrm{mcm}^{5} \mathrm{~s}^{2} \mathrm{U}\right)$, and the corresponding tRNAs decode in the split codon boxes. In contrast, $t R N A$ Gly and tRNA $_{U C G}^{\mathrm{Arg}}$, which contain $\mathrm{mchm}^{5} \mathrm{U}$, decode in family codon boxes. Therefore, one may speculate that the hydroxylation of $\mathrm{mcm}^{5} \mathrm{U}$ into mchm ${ }^{5} \mathrm{U}$ improves the reading of non-cognate codons; a typical feature of wobble uridine modifications found on family box tRNAs ${ }^{3,4}$. This ability may be of particular importance in higher eukaryotes, for example, mammals, which consist of a wide diversity of specialized cells, such as elongated neurons. Here, protein translation often occurs at discrete locations ${ }^{28}$, where low local concentrations of certain isoacceptors could necessitate the extensive use of non-cognate tRNAs in decoding.

Stereoisomers are frequently formed by epimerization, and one could envision $(R)-\mathrm{mchm}^{5} \mathrm{U}$ being formed by epimerization of $(S)-\mathrm{mchm}^{5} \mathrm{U}$, rather than by hydroxylation of $\mathrm{mcm}^{5} \mathrm{U}$. However, our presented data strongly argue against this notion, and predict an unknown oxygenase, OxX, for generation of $(R)-\mathrm{mchm}^{5} \mathrm{U}$ from $\mathrm{mcm}^{5} \mathrm{U}$. First, the observation that $(R)-\mathrm{mchm}^{5} \mathrm{U}$, but not $(S)-\mathrm{mchm}^{5} \mathrm{U}$, is present in the $\mathrm{KI}\left(M \mathrm{~T}^{+}\right)$mice indicates that the former

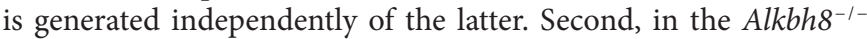

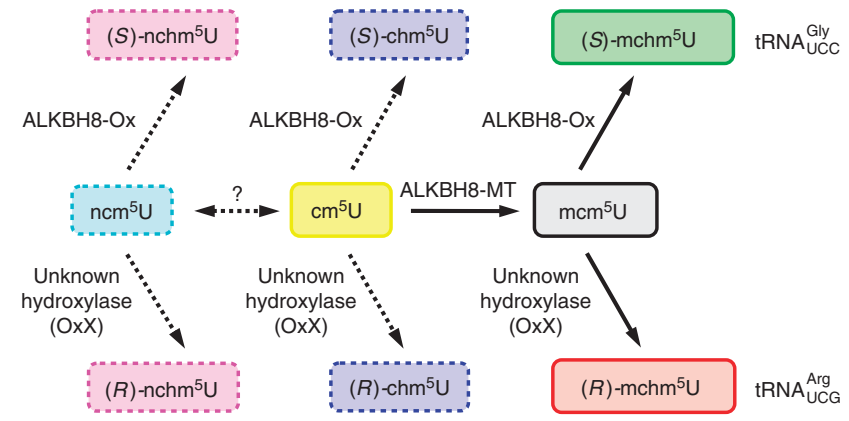

Figure 5 | Model for the generation of mchm $^{5} \mathrm{U}$ modifications in tRNA Gly and TRNA $\mathbf{U C C}$. The model depicts the roles of ALKBH8 and the putative oxygenase OxX in the formation of (S)-mchm ${ }^{5} U$ and $(R)-m c h m^{5} U$ in tRNA $\cup$ Gly and tRNA $\mathrm{Arg}$, respectively (indicated by solid lines in arrows and borders). Dashed lines in arrows and borders indicate reactions and modifications, respectively, that occur in TRNA $\mathrm{UCC}_{\mathrm{CC}}^{\mathrm{Gly}}$ and tRNA $\mathrm{ACG}$ when the $\mathrm{ALKBH} 8$ function is perturbed. The colour coding of borders corresponds to that of bar diagrams in Figure 2. mice, in which the ALKBH8-Ox and ALKBH8-MT functions are both absent, total tRNA contained $n \mathrm{nhm}^{5} \mathrm{U}$ (which is absent from WT tRNA). This presence of $n \mathrm{nh}^{5} \mathrm{U}$ may be explained by hydroxylation of accumulated $n \mathrm{~cm}^{5} \mathrm{U}$ by the enzyme OxX, which normally catalyses the 2 hydroxylation of $\mathrm{mcm}^{5} \mathrm{U}$ into $(R)$ - $\mathrm{mchm}^{5} \mathrm{U}$. Future studies will investigate various oxygenases, including ALKBH proteins of unknown function, as candidates for the OxX function.

Recently, the mammalian Tet proteins (Tet1, Tet2 and Tet3) were shown to be DNA hydroxylases converting epigenetic 5-methylcytosine $(5 \mathrm{mC})$ modifications into $5 \mathrm{hmC}^{8,29}$. Interesting analogies exist between ALKBH8 and the Tet proteins, as they all are 2OG/ $\mathrm{Fe}(\mathrm{II})$ oxygenases acting on nucleic acids, and as they hydroxylate corresponding positions in $\mathrm{mcm}^{5} \mathrm{U}$ and $5 \mathrm{mC}$, respectively. Conceivably, other ALKBH proteins may also catalyse reactions resulting in hydroxylation (rather than demethylation) of macromolecules.

\section{Methods}

Total tRNA and isoacceptor isolation. Total tRNA from S. cerevisiae and calf liver was purchased from Roche and Novagen, respectively. Total tRNA was purified from Mus musculus (liver), A. mellifera (head and thorax of worker bees), C. elegans, A. thaliana and S. pombe by using a RNA/DNA Maxi Kit (QIAGEN). Individual tRNA $\mathrm{GCC}_{\mathrm{Cly}}$ and tRNA $\mathrm{Arg}$ isoacceptors were purified from total tRNA using 3'biotinylated oligonucleotides (Supplementary Table S1), as previously described ${ }^{22}$.

Synthesis of $\mathbf{~ m c h m}^{5} \mathbf{U}$ nucleoside standards. $(R)$ - and $(S)-\mathrm{mchm}^{5} \mathrm{U}$ were obtained by a previously reported three-step procedure ${ }^{30}$. The structure and homogeneity of these nucleosides derivatives were confirmed by nuclear magnetic resonance and mass spectrometry.

LC-MS/MS of nucleosides. The analysis was performed essentially as described ${ }^{22}$. Briefly, tRNA was enzymatically digested to nucleosides ${ }^{31}$, which were separated by reverse phase high-performance liquid chromatography on a Zorbax SB-C18 column, using a mobile phase consisting of $0.1 \%$ formic acid and a gradient of $5-50 \%$ methanol. Online mass spectrometry detection was performed using an Applied Biosystems/MDS Sciex 5000 triple-quadrupole mass spectrometer (Applied Biosys tems Sciex) with TurboIonSpray probe operating in positive electrospray ionization mode. The nucleosides were monitored by multiple reaction monitoring using the nucleoside to base ion mass transitions $317.2 \rightarrow 185.1\left(\mathrm{mcm}^{5} \mathrm{U}\right), 333.2 \rightarrow 201.1$ $\left(\mathrm{mchm}^{5} \mathrm{U}\right), 303.2 \rightarrow 171.1\left(\mathrm{~cm}^{5} \mathrm{U}\right), 319.2 \rightarrow 187.1\left(\mathrm{chm}^{5} \mathrm{U}\right), 302.2 \rightarrow 170.1\left(\mathrm{ncm}^{5} \mathrm{U}\right)$ $318.2 \rightarrow 186.1\left(\mathrm{nchm}^{5} \mathrm{U}\right), 268.2 \rightarrow 136.1(\mathrm{~A}), 244.2 \rightarrow 112.1(\mathrm{C}), 284.2 \rightarrow 152.2(\mathrm{G})$ and $245.2 \rightarrow 113.1(\mathrm{U})$. Quantification was performed by comparison with pure nucleoside standards run in between the samples. Nucleoside standards for $\mathrm{ncm}^{5} \mathrm{U}$ and $n c h m^{5} \mathrm{U}$ were generated from $\mathrm{mcm}^{5} \mathrm{U}$ and $\mathrm{mchm}^{5} \mathrm{U}$ as previously described ${ }^{32}$, whereas $\mathrm{cm}^{5} \mathrm{U}$ and $\mathrm{chm}^{5} \mathrm{U}$ were generated by mild alkaline hydrolysis of $\mathrm{mcm}^{5} \mathrm{U}$ and $\mathrm{mchm}^{5} \mathrm{U}$ (ref. 22). The fragmentation pattern of the nucleoside standards was in accordance with theoretical predictions, and has been included in Supplementary Figure S13.

MALDI-TOF mass spectrometry. tRNA isoacceptors were digested with RNase T1 (Ambion) and samples prepared for mass spectrometry as previously described ${ }^{22}$. MALDI (matrix-assisted laser desorption/ionization) mass spectrometry was performed on a Perseptive Voyager STR instrument detecting positive ions in reflector TOF geometry. Spectrum processing was done with the MoverZ software (Genomic Solutions). MALDI tandem mass spectrometry was performed on a Waters QTOF Ultra MALDI instrument (Waters) with sample preparation as described above. The spectra were recorded in positive ion mode and processed with the supplied MassLynx software; additional details have been published previously ${ }^{33}$.

Gene-targeted mice. Gene-targeting strategy and screening method to verify homologous recombination are presented in Supplementary Figures S2-S5.

Expression of recombinant proteins. Human ALKBH8 (aa 1-664), or its MT domain (ALKBH8-MT; aa 352-664), both in complex with human TRM112, and its AlkB domain (ALKBH8-Ox; aa 1-354) were purified as amino-terminal 6xHistagged protein from $E$. coli as previously described ${ }^{22}$.

Enzymatic treatment of tRNA. tRNA $(5-10 \mu \mathrm{g})$ was incubated with recombinant protein $\left(100 \mathrm{pmol}\right.$, if not indicated otherwise) for $30 \mathrm{~min}$ at $37^{\circ} \mathrm{C}$ in a $50 \mu \mathrm{l}$ reaction mixture containing $25 \mu \mathrm{M} S$-adenosyl-L-methionine, $50 \mathrm{mM}$ Tris- $\mathrm{HCl} \mathrm{pH} 7.5$, $25 \mathrm{mM} \mathrm{KCl}_{2} 25 \mathrm{mM} \mathrm{NH}_{4} \mathrm{Ac}, 0.5 \mathrm{mM} \mathrm{MgCl}_{2}, 2 \mathrm{mM}$ ascorbic acid, $100 \mu \mathrm{M}$ 2-oxoglutarate, $40 \mu \mathrm{M} \mathrm{FeSO}_{4}, 10 \mathrm{U}$ RNase inhibitor and then precipitated with 1 volume of isopropanol in the presence of $1 \mathrm{M} \mathrm{NH}_{4} \mathrm{Ac}$ and $10 \mu \mathrm{g}$ of glycogen. Incubations with ALKBH8 and ALKBH8-MT were performed in the presence of the co-expressed and co-purified accessory protein TRM112. Pellets were washed with $70 \% \mathrm{EtOH}$, dried and dissolved in $\mathrm{H}_{2} \mathrm{O}$. The samples were subjected to nucleoside analysis by LC-MS/MS. 
Codon-run reporter constructs. Short dsDNA fragments containing NcoI-specific DNA overhangs at both ends and harbouring ten successive, identical Lys, Gln, Arg or Gly codons were generated by annealing oligo pairs $\mathrm{AAA}_{10} \mathrm{~s}^{-\mathrm{AAA}} \mathrm{AA}_{10} \mathrm{as}, \mathrm{AAG}_{10^{-}}$

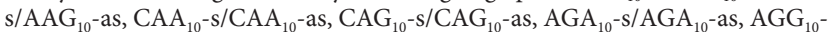
s/ $\mathrm{AGG}_{10}$-as, $\mathrm{GGA}_{10}{ }^{\mathrm{s}} / \mathrm{GGA}_{10}$-as or $\mathrm{GGG}_{10}$-s/GGG $10^{-}$as (Supplementary Table $\mathrm{S} 1$ ), respectively, and inserted downstream of the translation initiation codon of the firefly luciferase gene in pDualLuc-IRES3 using its $\mathrm{NcoI}$ site ${ }^{34}$. Subsequently, NotIHindIII fragments from pDualLuc-IRES3 or derivatives containing a codon-run insertion were transferred to pCMV-Script (Stratagene). Following this strategy, pDualLuc-LysAAA ${ }_{10}$, pDualLuc-LysAAG ${ }_{10}$, pDualLuc-GlnCAA 10 , pDualLucGlnCAG $_{10}$, pDualLuc-ArgAGA 10 , pDualLuc-ArgAGG 10 , pDualLuc-GlyGGA 10 and pDualLuc-GlyGGG ${ }_{10}$ were constructed (Fig. 4a).

In vivo translation efficiency assay. Primary mouse embryonic fibroblasts were maintained in DMEM (BioWhittaker) supplemented with 15\% fetal bovine serum (Saveen \& Werner) and $100 \mathrm{U}$ per ml penicillin and streptomycin. Cells were grown to $90 \%$ confluency and transfected using a MicroPorator MP-100 (Digital Bio Technology, NanoEnTek). Cells $\left(5 \times 10^{5}\right)$ were transfected with pDualLuc reporter plasmids using a pulse voltage of $1,350 \mathrm{~V}, 30 \mathrm{~ms}$ pulse width and a $100 \mu \mathrm{l}$ Gold Tip according to the manufacturer's instructions, and immediately after electroporation transferred to pre-warmed 12-well dishes containing DMEM without antibiotics. Cells were collected at $24 \mathrm{~h}$ post-transfection and the intracellular Fluc and Rluc activities were subsequently measured using a Luminometer (Turner Designs TD-20/20), using the Dual-luciferase Reporter Assay according to the manufacturer's protocol (Promega).

\section{References}

1. Agris, P. F. Decoding the genome: a modified view. Nucleic Acids Res. 32, 223-238 (2004)

2. Sprinzl, M. \& Vassilenko, K. S. Compilation of tRNA sequences and sequences of tRNA genes. Nucleic Acids Res. 33, D139-D140 (2005).

3. Johansson, M. J., Esberg, A., Huang, B., Björk, G. R. \& Byström, A. S. Eukaryotic wobble uridine modifications promote a functionally redundant decoding system. Mol. Cell Biol. 28, 3301-3312 (2008).

4. Nasvall, S. J., Chen, P. \& Björk, G. R. The modified wobble nucleoside uridine5 -oxyacetic acid in tRNAPro(cmo5UGG) promotes reading of all four proline codons in vivo. RNA 10, 1662-1673 (2004).

5. Huang, B., Johansson, M. J. \& Byström, A. S. An early step in wobble uridine tRNA modification requires the Elongator complex. RNA 11, 424-436 (2005).

6. Kalhor, H. R. \& Clarke, S. Novel methyltransferase for modified uridine residues at the wobble position of tRNA. Mol. Cell Biol. 23, 9283-9292 (2003).

7. Loenarz, C. \& Schofield, C. J. Expanding chemical biology of 2-oxoglutarate oxygenases. Nat. Chem. Biol. 4, 152-156 (2008).

8. Tahiliani, M. et al. Conversion of 5-methylcytosine to 5-hydroxymethylcytosine in mammalian DNA by MLL partner TET1. Science 324, 930-935 (2009).

9. Tsukada, Y. et al. Histone demethylation by a family of JmjC domaincontaining proteins. Nature 439, 811-816 (2006).

10. Aas, P. A. et al. Human and bacterial oxidative demethylases repair alkylation damage in both RNA and DNA. Nature 421, 859-863 (2003).

11. Falnes, P. O., Johansen, R. F. \& Seeberg, E. AlkB-mediated oxidative demethylation reverses DNA damage in Escherichia coli. Nature 419, 178-182 (2002).

12. Trewick, S. C., Henshaw, T. F., Hausinger, R. P., Lindahl, T. \& Sedgwick, B. Oxidative demethylation by Escherichia coli AlkB directly reverts DNA base damage. Nature 419, 174-178 (2002).

13. Gerken, T. et al. The obesity-associated FTO gene encodes a 2-oxoglutaratedependent nucleic acid demethylase. Science 318, 1469-1472 (2007).

14. Kurowski, M. A., Bhagwat, A. S., Papaj, G. \& Bujnicki, J. M. Phylogenomic identification of five new human homologs of the DNA repair enzyme AlkB. BMC Genomics 4, 48 (2003).

15. Duncan, T. et al. Reversal of DNA alkylation damage by two human dioxygenases. Proc. Natl Acad. Sci. USA 99, 16660-16665 (2002).

16. Westbye, M. P. et al. Human AlkB homolog 1 is a mitochondrial protein that demethylates 3-methylcytosine in DNA and RNA. J. Biol. Chem. 283, 25046-25056 (2008).

17. Ringvoll, J. et al. Repair deficient mice reveal $\mathrm{mABH} 2$ as the primary oxidative demethylase for repairing $1 \mathrm{meA}$ and $3 \mathrm{meC}$ lesions in DNA. EMBO J. 25, 2189-2198 (2006).

18. Nordstrand, L. M. et al. Mice lacking Alkbhl display sex-ratio distortion and unilateral eye defects. PLoS ONE 5, e13827 (2010).
19. Pan, Z. et al. Impaired placental trophoblast lineage differentiation in Alkbh1(-/ - ) mice. Dev. Dyn. 237, 316-327 (2008).

20. Sedgwick, B., Bates, P. A., Paik, J., Jacobs, S. C. \& Lindahl, T. Repair of alkylated DNA: recent advances. DNA Repair (Amst.) 6, 429-442 (2007).

21. Fu, D. et al. Human AlkB homolog ABH8 Is a tRNA methyltransferase required for wobble uridine modification and DNA damage survival. Mol. Cell Biol. 30, $2449-2459$ (2010).

22. Songe-Moller, L. et al. Mammalian ALKBH8 possesses tRNA methyltransferase activity required for the biogenesis of multiple wobble uridine modifications implicated in translational decoding. Mol. Cell Biol. 30, 1814-1827 (2010).

23. Kawakami, M., Takemura, S., Kondo, T., Fukami, T. \& Goto, T. Chemical structure of a new modified nucleoside located in the anticodon of Bombyx mori glycine tRNA2. J. Biochem. 104, 108-111 (1988).

24. Fu, Y. et al. The AlkB domain of mammalian ABH8 catalyzes hydroxylation of 5-methoxycarbonylmethyluridine at the Wobble position of tRNA. Angew. Chem. Int. Ed. Engl. 49, 8885-8888 (2010).

25. Andersen, T. E., Kirpekar, F. \& Haselmann, K. F. RNA fragmentation in MALDI mass spectrometry studied by H/D-exchange: mechanisms of general applicability to nucleic acids. J. Am. Soc. Mass Spectrom. 17, 1353-1368 (2006).

26. Begley, U. et al. Trm9-catalyzed tRNA modifications link translation to the DNA damage response. Mol. Cell 28, 860-870 (2007).

27. Dewez, M. et al. The conserved Wobble uridine tRNA thiolase Ctu1-Ctu2 is required to maintain genome integrity. Proc. Natl Acad. Sci. USA 105, 5459-5464 (2008).

28. Holt, C. E. \& Bullock, S. L. Subcellular mRNA localization in animal cells and why it matters. Science 326, 1212-1216 (2009).

29. Ito, S. et al. Role of Tet proteins in $5 \mathrm{mC}$ to $5 \mathrm{hmC}$ conversion, ES-cell selfrenewal and inner cell mass specification. Nature 466, 1129-1133 (2010).

30. Nawrot, B. \& Malkiewicz, A. The tRNA 'wobble position' uridines. III. The synthesis of 5-[S-methoxycarbonyl(hydroxy)metyl]uridine and its 2-thio analogue. Nucleosides Nucleotides 8, 1499-1512 (1989).

31. Crain, P. F. Preparation and enzymatic hydrolysis of DNA and RNA for mass spectrometry. Methods Enzymol. 193, 782-790 (1990).

32. Fissekis, J. D. \& Sweet, F. Synthesis of 5-carboxymethyluridine. A nucleoside from transfer ribonucleic acid. Biochemistry 9, 3136-3142 (1970).

33. Mengel-Jorgensen, J. \& Kirpekar, F. Detection of pseudouridine and other modifications in tRNA by cyanoethylation and MALDI mass spectrometry. Nucleic Acids Res. 30, e135 (2002).

34. van den Born, E., Posthuma, C. C., Gultyaev, A. P. \& Snijder, E. J. Discontinuous subgenomic RNA synthesis in arteriviruses is guided by an RNA hairpin structure located in the genomic leader region. J. Virol. 79, 6312-6324 (2005).

\section{Acknowledgments}

We thank H. Nilsen, R. Aamodt and I. Alseth for supplying biological material for tRNA isolation from C. elegans, A. mellifera and S. pombe, respectively. We are grateful to Cesilie G. Castellanos and Hege Wiksen for excellent technical assistance and animal care. We thank GenOway, Lyon, France, The Norwegian Transgenic Center (NTS) and the Centre for Comparative Medicine at Oslo University Hospital HF for the excellent services they provided. We thank Stefan Kernstock and Angela Ho for critical reading of the manuscript. This work was supported by grants from the FRIBIO and FUGE programs in the Research Council of Norway, as well as Grant PNRF-143-AI-1/07 from the Polish-Norwegian Research Fund (to A.K. and P.Ø.F.)

\section{Author contributions}

E.v.d.B, C.B.V., L.S.-M., V.L., G.F.L. and F.K. designed and performed experiments, and analysed data. G.L. synthesized nucleoside standards. H.E.K. and A.M. analysed data. A.K. and P.Ø.F. initiated the study, designed experiments, analysed data and wrote the manuscript. All authors discussed the results and commented on the manuscript.

\section{Additional information}

Supplementary Information accompanies this paper at http://www.nature.com/ naturecommunications

Competing financial interests: The authors declare no competing financial interests. Reprints and permission information is available online at http://npg.nature.com/ reprintsandpermissions/

How to cite this article: van den Born, E. et al. ALKBH8-mediated formation of a novel diastereomeric pair of wobble nucleosides in mammalian tRNA. Nat. Commun. 2:172 doi: 10.1038/ncomms1173 (2011). 